\title{
KrF Excimer Laser Micromachining of Silicon for Micro- Cantilever Applications
}

\section{A.F.M. Anuar ${ }^{1 *}$, Y. Wahab, H. Fazmir, M. Najmi, S. Johari, M. Mazalan, N.I.M. Nor, M.K. Md Arshad}

1 Advanced Multi-Diciplinary MEMS-Based Integrated NCER Centre of Excellent (AMBIENCE), School of Microelectronic, Universiti Malaysia Perlis, Malaysia. fitrianuarm@yahoo.com ; yufridinwahab@unimap.edu.my

* Author to whom correspondence should be addressed; E-Mail: fitrianuarm@yahoo.com Tel.: +604-9852557; Fax: +604-9852558.

Published: 1 June 2014

\begin{abstract}
The conventional photolithography of crystalline silicon techniques is limited to two-dimensional and structure scaling. This can be overcome by using laser micromachine, a technique capable of producing three-dimensional structure and simultaneously avoiding the needs for photomasks. In this paper, we report on the use of RapidX-250 excimer laser micromachine with $248 \mathrm{~nm} \mathrm{KrF}$ to create in-time mask design and assisting in the fabrication of micro-cantilever structures. Three parameters of the laser micromachine used to aid the fabrication of the micro-cantilever have been investigated; namely the pulse rate (i.e. number of laser pulses per second), laser energy and laser beam size. Preliminary results show that the $35 \mathrm{um}$ beam size and $15 \mathrm{~mJ}$ of energy level is the most effective parameter to structure the desired pattern. The parallel lines spacing of the structure can be reached up to 10 um while cutting, holes drilling and structuring the cantilever using the laser beam can be accomplished to as low as 50 um in dimension.
\end{abstract}

Keywords: Excimer laser; KrF; Silicon Micromachine; Micro-Cantilever

\section{Introduction}

The crystalline silicon wafer is a thin slice of semiconductor material that is used in microdevices and integrated circuit in the electronics fabrication. The microelectronic devices use silicon wafer as substrate and have many fabrication steps include etching, diffusion, materials deposition and photolithography process. The conventional photolithography 
process is the most crucial step in the microfabrication process since the design is transferred to the device by etching process which requires various specific parameters and rules. Nowadays, microelectronic devices are required to be small, low in cost, high-density integration in single die and fast to produce. In order to fabricate the complex threedimensional structure without a mask, micromachine with laser ablation is the best option. In the year 1982, materials like polymer has been widespreadly investigated by researchers using excimer laser radiation [1]. Depending on processing materials, every laser pulse has their own merits and demerits. In this research, Resonetics laser micromachine is used for the crystalline silicon characterization, with Krypton fluoride $(\mathrm{KrF})$ as the particular gas needs to generate excimer laser.

\section{Excimer Laser Micromahine}

The 248nm of wavelength is a deep laser which is commonly used in semiconductor industrial manufacturing [2]. The RapidX 250 series of $\mathrm{KrF}$ excimer micromachine is used in this research as a part of an excimer laser system. The most important aspects provided by laser pulsed micromachining includes good quality in high resolution, high precision, high process speeds, low thermal damage and applicable to many materials [3]. Besides, it also needs few processing steps, highly flexible Computer Numerical Control (CNC) programming depending on prototyping shapes, no larger cleanroom facilities and less hazardous to human substances. The system used in this research is shown in Figure 1 which consists of mechanical parts and X-Y stages orientation controlled by user friendly software.

Figure 1. KrF excimer laser micromachine system; (a) Physical structure of Resonetics laser micromachine, (b) Laser beam circulation and internal hardware system.

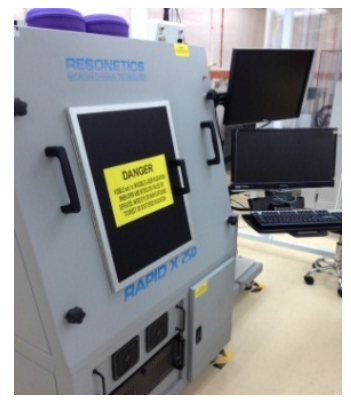

(a)

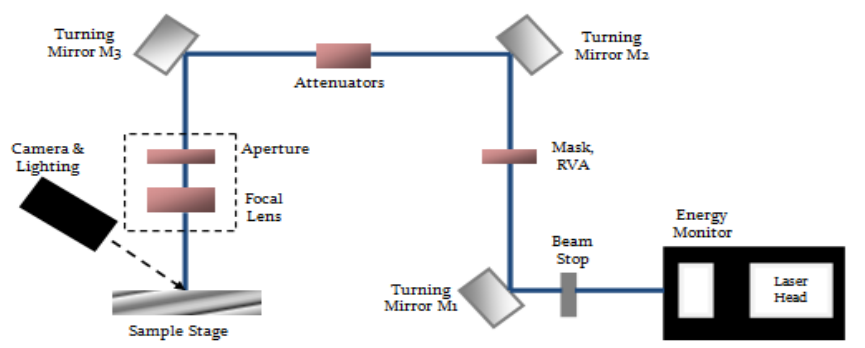

(b)

The frequency of the laser emission is sufficiently energetic to break the chemical bonds of most materials [4]. As shown in Figure 2, laser pulses are bombarded on the silicon surface. In this section, photochemical process occurs where photon, which is directed laser energy is absorbed by the silicon surface.. Thus, heat is generated and leads to the photothermal process. The unique characteristic of the excimer laser with 248nm optical wavelength provide high resolution ( $\sim \mathrm{um}$ ) features on the target surface, while the etching process on a silicon substrate within fluential power of $15 \mathrm{~mJ}$ will create $0.1 \mathrm{um}$ to $1 \mathrm{um}$ average depth into the sample [5]. 
Figure 2. Interaction between laser and silicon.

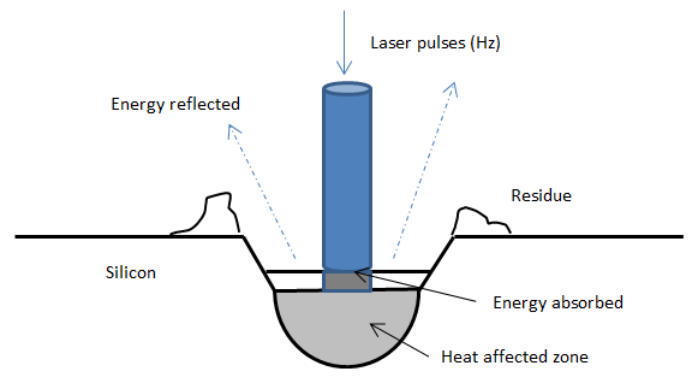

\section{Silicon Micromachining}

Crystalline silicon ( $\mathrm{Si}$ ) is a common semiconductor material that is widely used nowadays for microelectronic fabrications. In order to find the best recipes for excimer laser ablation of silicon materials, a set of experiments with different parameters were conducted. The main parameter investigated in this research is laser energy $(\mathrm{mJ})$ which is laser beam energy level that are supplied to form laser beam. The number of laser pulses in second is a part of important criteria for excimer laser experiment. The maximum number of laser pulses provide by the RapidX-250 laser micromachine is 300 pulses. The number of pulses will be determining the laser line pattern either in gross or smooth line. Besides, etch rate in $\mu \mathrm{m} / \mathrm{shot}$ is defined as average etched depth by each laser shot. The other parameter required in this research is the laser beam size aspect ratio which is defines as Rectangular Variable Aperture (RVA) in milimeters unit.

\subsection{Etch Depth}

The micromachine patterns on a silicon substrate were observed using high power microscope (HPM) with various lens magnification. The tested pattern with different pulse frequencies on a single line were successfully imaged on the sample shown in Figure 3. The experiments were carried out with unchanged parameters unless different pulse repetition on the same line is required. Figure 3 illustrates the 9.08um of the etch depth with 30 times of pulse scanning repetition.

Figure 3. Etch rate characteristics; (a) Etch depth for 30 times lasers scanning, (b) Relations between number of laser scanning and etch depth.

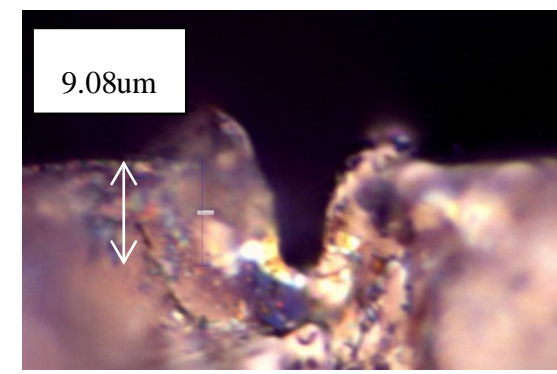

(a)

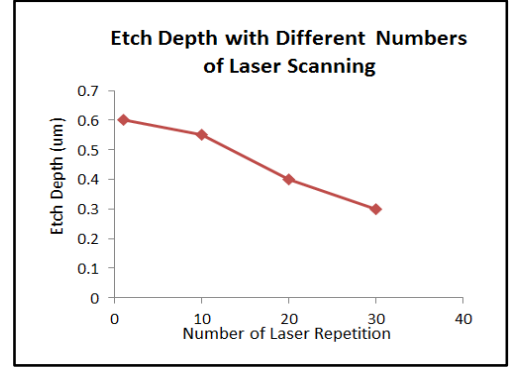

(b) 


\subsection{Rectangular Variable Aperture (RVA)}

The size of laser ablation illustration can be obtained by comparing the width geometries of the laser sizes. Figure 5 compares $0.5 \mathrm{~mm}, 1.0 \mathrm{~mm}$ and $2.0 \mathrm{~mm}$ of RVA with constant energy supplied. The double beam width size of RVAs is shown in Figure 5 (a) and Figure 5 (b).

Figure 5. Laser ablation by differences RVA; (a) $0.5 \mathrm{~mm}$ of RVA, (b) $1.0 \mathrm{~mm}$ of RVA, (c) $2.0 \mathrm{~mm}$ of RVA.

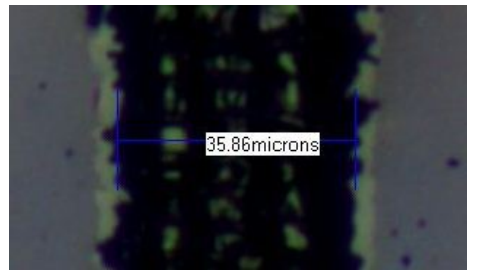

(a)

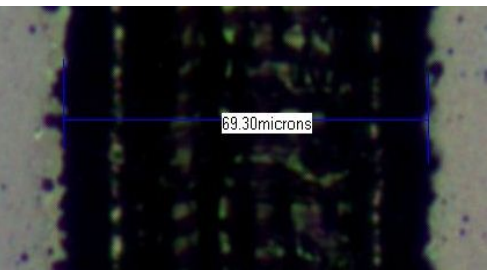

(b)

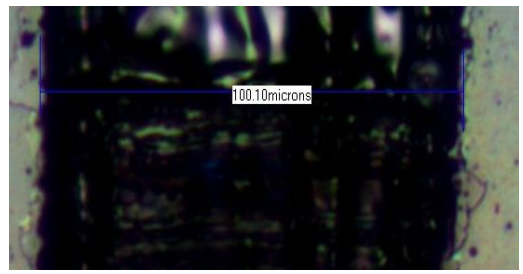

(c)

\subsection{Power}

Energy level is important for design structures which have varying effects on depth and surface profiles. The precise desired pattern can be achieved by ensuring the sufficient power needs by the target. The range of the $\mathrm{KrF}$ excimer laser micromachining laser energy can be varied from $1 \mathrm{~mJ}$ to $18 \mathrm{~mJ}$. The surface morphology of the sample tested using laser power at $12 \mathrm{~mJ}, 15 \mathrm{~mJ}$ and $18 \mathrm{~mJ}$ are shown in Figure 6 . Based on these results, it can be concluded that $15 \mathrm{~mJ}$ is the suitable energy for laser to fire up the structure on the silicon substrate.

Figure 6. Surface morphology with difference energy level;

(a) $12 \mathrm{~mJ}$, (b) $15 \mathrm{~mJ}$, (c) $18 \mathrm{~mJ}$.

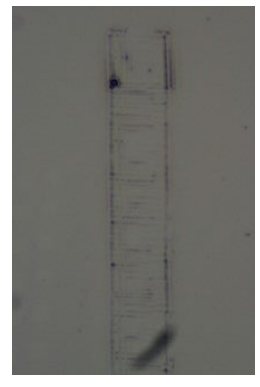

(a)

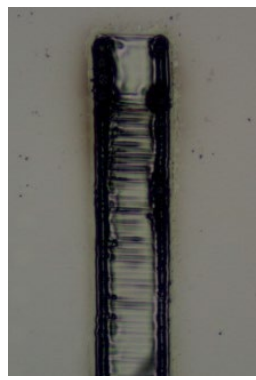

(b)

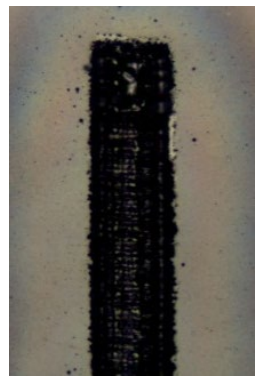

(c)

\subsection{Pulse Number}

The smoothness and planarization of the silicon surface is also affected by the pulse number of the laser. In this research, we found that using low number of laser pulse will provide high roughness on the depth of firing structure. Thus the zig-zag pattern will be likely produce by a laser beam. Figure 7 shows the top surface of the sample with different number of laser pulse. The variables of pulse number can be adjusted in the range of 1 to 300 pulses. 
Figure 7. Differences in pulse number;

(a) 10 pulses, (b) 50 pulses, (c) 100 pulses

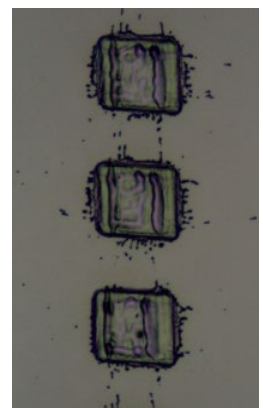

(a)

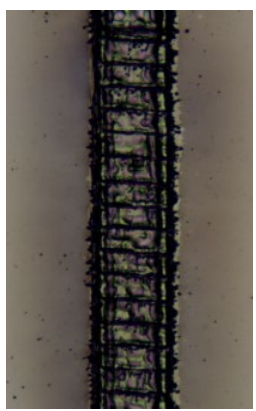

(b)

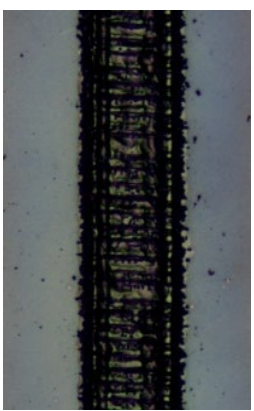

(c)

\section{Silicon Microcantilever}

Silicon microcantilever development by excimer laser micromachining is faster in terms of time and no wet chemical needed for the overall process. In this research, silicon substrate with the thickness of 160 um is prepared for the microcantilever development. Figure 8 shows the distance resolution for 50um of parallel lines. Laser energy at $18 \mathrm{~mJ}$ and $0.5 \mathrm{~mm}$ of RVA affected the 10um resolution between these lines. The beam size needs to be considered because it will strike the total parameter of the design.

Figure 8. Parallel lines distance resolution

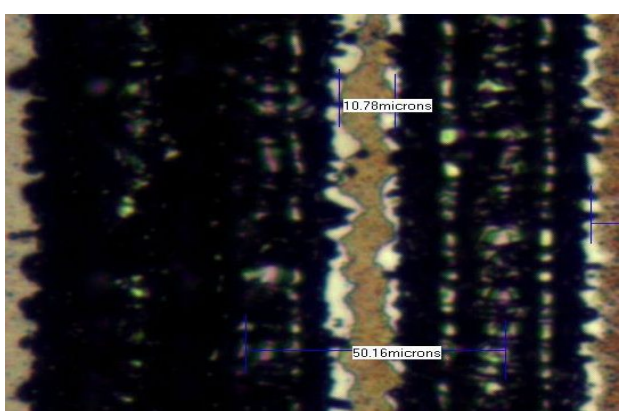

Scanning pulse at 250 pulses, $15 \mathrm{~mJ}$ of laser energy and $0.5 \mathrm{~mm}$ RVA were programmed to obtain a high quality of microcantilever structure. Cantilever dimension was designed in AutoCAD with $1.0 \mathrm{~mm} \times 0.4 \mathrm{~mm}$. In Figure 9 (b), microcantilever structure has been produced by 290 times of laser beam scanning. The experimental result shows the dimension of microcantilever of 990um $\mathrm{x} 350 \mathrm{um}$ for the length and width respectively. It is demonstrated that the features of pulse etching depth can be defined as $0.5 \mathrm{um}$ per scan for silicon substrate. Low power and small beam size are used in the formation of microcantilever because of the lower edge roughness and heat radiation on the side of the line. These factors can interfere the characteristic of the deposited material on the substrate if the microcantilever were to be used as sensors or actuators. 
Figure 9. Microcantilever formation by excimer laser micromachine;

(a) Mask design in millimeter, (b) Microcantilever top view.

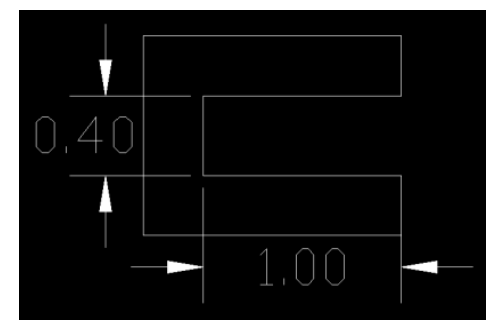

(a)

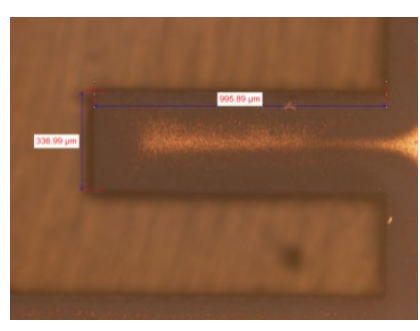

(b)

\section{Conclusion}

Microcantilevers are the most simplified sensors and actuators for MEMS based devices. This paper demonstrated the $\mathrm{KrF}(248 \mathrm{~nm})$ excimer laser micromachine of crystalline silicon for microcantilever structure. Silicon thickness of 160um was prepared for microcantilever formation and the optimum parameter is programmed in the computerize operations process. The maximum laser energy will affect the edge roughness and heat radiation on the side of the line. This is probably related to the presence of thermal radiation and laser beam aperture in the laser formation process. The laser repeatable and beam aperture also affected the roughness of the microcantilever structure. The desired structure can be reached with $15 \mathrm{~mJ}$, 250 pulses, $0.5 \mathrm{~mm}$ of the power, scanning laser and RVA respectively. Physical characteristic by HPM reveals that laser ablation with these parameters can produce the optimum cantilever structure.

\section{Acknowledgments}

It is a pleasure to thank Prof Assoc Dr Yufridin Wahab, Mazlee Malazan and Dr Shazlina Johari for many useful discussions according to the research topic. This work was supported by a grant in AMBIENCE, School of Microelectronic Engineering, UniMAP, Perlis, Malaysia.

\section{Conflicts of Interest}

The authors declare no conflict of interest.

\section{References and Notes}

[1] Y Kawamura, K Toyoda and S Namba, 'Effective deep ultraviolet photoetching of polymethyl methacrylate by an excimer laser’, Appl Phys Letts 40, 374, (1982)

[2] Laser Micromachining Group Laser Systems Group, "Laser Micromachining Seminar.”

[3] Resonetics Micromachining Technology, Maestro series Excimer laser workstation, Technical reference, Rev. B, (2002).

[4] A. S. Holmes, J. E. a. Pedder, and K. L. Boehlen, "Advanced laser micromachining processes for MEMS and optical applications,” vol. 6261, p. 62611E-62611E-9, May 2006.

[5] Y. Wahab and N. A. Bakar, "MEMS Biomedical Sensor for Gait Analysis.” 